Stat Med. 2010 August 30; 29(19): 1985-1997. doi:10.1002/sim.3933.

\title{
WHAT IS EVIDENCE?
}

\author{
Sharon-Lise T. Normand ${ }^{1,2}$ and Barbara J. McNeil ${ }^{1,3}$ \\ ${ }^{1}$ Harvard Medical School, Department of Health Care Policy, Boston, U.S.A. \\ ${ }^{2}$ Harvard School of Public Health, Department of Biostatistics, Boston, U.S.A. \\ ${ }^{3}$ Department of Radiology, Brigham \& Womens Hospital,3 Boston, Massachusetts U.S.A.
}

\section{SUMMARY}

The assumption that comparative effectiveness research will provide timely, relevant evidence rests on changing the current framework for assembling evidence. In this commentary, we provide the background of how coverage decisions for new medical technologies are currently made in the United States. We focus on the statistical issues regarding how to use the ensemble of information for inferring comparative effectiveness. It is clear a paradigm shift in how clinical information is integrated in real-world settings to establish effectiveness is required.

\section{Keywords}

evidentiary evaluation; multiple outcomes and comparisons; hierarchical Bayesian models; Bayes factors; posterior predictive probability

\section{INTRODUCTION}

The current paradigm for integrating clinical information in real-world settings to establish whether benefits outweigh risks is out-dated. Divergence from this paradigm involves the recognition that randomized controlled trials that often serve as the basis for new technology approval are small and short-term, and post-market studies are often voluntary and difficult to implement. These problems have become increasingly important over the last decade because technology is changing at a rapid pace, therapies are utilized outside their intended populations, and more representative groups of patients are likely to have differential responses to the same therapy.

In their paper, Tunis and colleagues identify several areas of improvement to provide more and better evidence of what works. Their thesis, and that of many others, relies on the premise that comparative effectiveness research will provide "timely, relevant evidence." While we agree with their general premise, the framework for determining what constitutes evidence and how it can be obtained in a timely manner motivates our commentary. We begin with a review of how coverage decisions for new medical technologies are currently made in the United States. We then describe the statistical methods for aggregating the ensemble of information to make inferences.

Corresponding Author: Sharon-Lise Normand, Department of Health Care Policy, Harvard Medical School, 180 Longwood Avenue, Boston, MA 02115 U.S.A. sharon@hcp.med.harvard.edu; Phone: 617-432-3260; Fax: 617-432-0173. 


\section{HOW ARE DECISIONS MADE?}

When a new medical technology is approved or cleared for use by the U.S. Food and Drug Administration, the manufacturer of the technology typically follows with a request to the Center for Medicare and Medicaid Services (CMS) for either a local coverage decision or a national coverage decision. This is a decision on whether CMS will provide coverage for the new technology. The CMS is the U.S. federal agency that administers the Medicare, Medicaid, and States Childrens Health Insurance Programs, and as such, is the largest health insurer in the United States. The majority of requests for coverage by CMS are for local decisions because a denial resulting from a national request has much more serious consequences than does one from a local request. National coverage decisions made by the CMS have substantial impact on the use of new technologies because other insurers frequently follow suit.

In some cases, the CMS will seek guidance from the Medicare Evidence Development \& Coverage Advisory Committee (MedCAC), an independent advisory committee which was established in 1998. The MedCAC judges the strength of the available evidence including medical literature, technology assessments, and data on effectiveness and appropriateness of technologies and services, and makes recommendations to the CMS based on that evidence [1]. Table 1 briefly summarizes the evaluation process used by the MedCAC in its deliberations for determining the effectiveness of new therapies[2]. While the MedCAC evaluation process focuses on the CMS population, other protocols, sometimes quite similar to those used by CMS, are used by private health insurance plans for their specific populations. All find the need to consider data from multiple different sources and multiple types of experimental designs.

The original evaluation approach for national requests for CMS was associated with binary decisions by the MedCAC panel, i.e., the new clinical service or technology did or did not meet evidentiary requirements for coverage. Several years later the process was changed. Now the panel is asked to provide, among other things, confidence ratings using a 1 to 5 scale on the adequacy of the evidence to draw conclusions about health outcomes and using the same scale, to provide the likelihood that the new technology actually improved health outcomes.

This evaluation process involves a series of challenging statistical issues. Step (1) in Table (1) requires assessment of the quality of the evidence in order to weigh it. A long literature exists, generally in the area of meta-analysis, regarding the difficulties with creating quality indices and using them as weighting factors to average across different sources of evidence[3,4]. Tunis and colleagues suggest that more involvement of patients, consumers, clinicians, and payers in key phases of CER development and implementation. With wide variation among researchers in determining quality scores, we expect even more variation across such diverse groups of individuals. It is not even clear how the CMS weights the information from the MedCAC: how does the CMS combine the panelists confidence ratings with their ratings on the likelihood the technology improved health outcomes to arrive at a coverage decision. A casual review of recent CMS assessments and responses by the MedCAC to questions regarding confidence in the quality of the data indicates wide variation. About $60 \%$ of the time CMS issues favorable decisions and in the majority of cases reviewed, the strength of the evidence is considered poor (see the discussion in [6])

Step (2) in Table (1) involves a comparison of, potentially, many existing therapies to the new therapy on the basis of many different outcomes. Two problems immediately emerge: how to analyze multiple outcomes and how to compare the new technology with the current existing therapies. The increasing use of multiple outcomes to comprehensively assess a 
treatment or clinical service effect arises because manufacturers wish to demonstrate clinical effectiveness on multiple outcomes. While there are statistical methods to deal with the correlation induced among multiple outcomes (see [5] and references therein), they are rarely utilized. More often, a composite endpoint is used by typically weighting all endpoints equally. In the assessment of coronary stents, for example, the composite of death, heart attack, stroke, or repeat revascularization is the defacto primary outcome. Importantly, there may be multiple alternative treatments rather than a single alternative, or there may be a lack of consensus regarding if standard exists.

A daunting problem facing the MedCAC and the CMS is the need for extrapolation of the treatment benefit to their population. The extrapolation problem is significant based on the lack of clinical trial participation of key subgroups of importance to the CMS. As an example, researchers have compared the fraction of patient subgroups participating in clinical trials to the same fraction as potentially eligible in the community. One study [7] reported that the percentage of women in randomized trials of acute coronary syndromes published between 1991 and 2000 was $25 \%$ despite comprising $43 \%$ of all patients having a heart attack in the U.S. In the same article, the authors found that trial enrollment of patients aged 75 years or greater was 9\% while among all patients with heart attack in the U.S., 37\% are aged 75 or greater. Another study[8] has shown similar results for clinical trials in cancer.

\section{WHAT IS EVIDENCE?}

Because scientific evidence is the accumulation of information to support or refute a theory or hypothesis, we focus on the statistical problem of how to use the ensemble of information for inferring comparative effectiveness. Steps (3) and (4) in Table (1) involve inferring treatment benefit, often within a population that has not been studied and comparing the treatment benefit to multiple alternatives on possibly multiple outcomes. We assume that information is available for several outcomes measured across a variety of treatments and patient cohorts, and the goal is to infer effectiveness of the new technology compared to other approved technologies. The information may come from randomized trials and observational studies, and not all outcomes-treatment-cohorts may be observed. The idea is that assembling all the available information may reduce uncertainty about the effectiveness of the new technology compared to existing technologies in a setting where we believe particular relationships exist but are uncertain about their relevance. The natural approach in this setting is through a Bayesian hierarchical model. Let $Y_{i j k t m}$ denote the number of subjects improving in study $i$ of type $j$ (i.e., randomized trial, observational study) for patient subgroup $k$ (i.e., old, young), receiving technology $t$ for outcome $m$.

$$
y_{i j k t m} \mid p_{i j k t m} \sim \operatorname{Binomial}\left(n_{i j k t m}, p_{i j k t m}\right) ; \theta_{i j k t m}=\log \left(\frac{p_{i j k t m}}{1-p_{i j k t m}}\right)
$$

$$
\theta_{i j k t m} \mid \zeta \sim \operatorname{Normal}\left(\alpha_{m}+\beta_{t}+\gamma_{m t}+\delta_{j}+a_{i}+b_{j}+c_{k(i)}+d_{t(i)}+e_{m(i)}, \sigma_{i j k t m}\right)
$$

$\left(\zeta=\left\{\alpha_{m}, \beta_{t}, \gamma_{m t}, \delta_{j}, a_{i}, b_{j}, c_{k(i)}, d_{t(i)}, e_{m(i)}, \sigma_{i j k t m}\right\}\right.$ and where $\alpha_{m}$ represents the baseline logodds for outcome $\alpha_{m} ; \beta_{t}$ is the log-odds of the effect of technology $t$ for the average study and average outcome; $\delta_{j}$ denotes the type of study design; $\gamma_{m t}$ is the deviation from the average effect of technology $t$ on outcome $m$, and the terms $a_{i}, b_{j}, c_{k(i)}, d_{t(i)}$, and $e_{m(i)}$ are random effects for study, study design, cohort within study, technology within study, and outcome within study. The only fixed factor-level effects assumed are $\alpha_{m}, \beta_{t}, \gamma_{m}$, and $\delta_{j}$. The general model above is attributed DuMouchel[9] who has pioneered Bayesian methods for 
combining information. The model uses the variance components to model the expected correlations among the findings reported from the same study. We have assumed for ease of discussion that all outcomes are binary but mixed outcomes are accommodated.

The model reflects the ideas that when considering the effectiveness of a technology within a broad population, (1) evidence from non-randomized studies will be relevant and needs to be considered simultaneously with evidence from randomized studies; and (2) outcomes from similar technologies in similar population are likely related. The weights used to pool the study estimates are determined by the amount of within- and between-study variability that exists and what we view as exchangeable. In the model above, we have assumed exchangeability among outcomes within treatments and study designs. For example, if there are 5 observational studies and 3 randomized studies reporting on one outcome, then the individual observational study-level log-odds would be shrunk towards the mean log-odds ratios for the 5 observational studies and the individual randomized study-level estimates would be shrunk towards the mean log-odds of the 3 randomized studies. The shrinkage factor for the former is the within-observational study variation divided by the sum of the between-observational study variation and the within-observational study variation. The overall effect for the effectiveness of the new technology from the ensemble of observational and randomized studies is then calculated as another weighted estimate of the overall shrunken estimate from the observational studies and the overall shrunken estimate from the randomized studies. In a similar fashion, estimates for specific cohorts can be obtained. Studies not reporting a particular outcome can be viewed as having missing data for that outcome.

Two key features of this approach have not been fully exploited and are directly related to the topic of this commentary. First, two technologies can be compared by integrating over the parameter space to obtain posterior distribution for the appropriate function of parameters, i,e, $P\left(\beta_{t}-\beta_{t^{*}}>\Delta \mathrm{l} m\right.$, data $)$ for $t \neq t^{*}$. Moreover, if two technologies have not been directly compared, then through additional assumptions (those found in network metaanalysis[10]), posterior predictive distributions of differences in outcomes or of particular effect sizes can be calculated.

Second, Bayes factors can be computed to provide quantitative measures of evidence. While numerous articles have been written discussing the problems with p-values (see [11] and references therein), the health care field remains deeply entrenched with finding statistically significant results. In theory, Bayes factors could be computed for the comparison of new technologies with existing technologies and used for a basis for decision-making.

\section{DISCUSSION}

The empirical basis of comparative effectiveness research relies upon data accumulated from multiple diverse settings and designs, and will involve multiple outcomes and, depending on the current standard of care, multiple comparisons. Because of the increasing frequency to analyze combined data sources, statistical tools that can handle the complexity of such the data will require further enhancement and experience. The model complexity will require acquiring evidence supporting model assumptions and tools to assess robustness of conclusions to departures from the assumptions made. Importantly, more real-world applications are needed to test their usability and validity.

\section{Acknowledgments}

Funding: Dr. Normand was supported, in part, by grant R01-MH54693 from the National Institute of Mental Health. 


\section{References}

1. [January 8, 2010]. See http://www.cms.hhs.gov/FACA/02_MEDCAC.asp

2. [January 8, 2010]. See http://www.cms.hhs.gov/FACA/Downloads.recommendations.pdf

3. Greenlander S, O'Rourke K. On the bias produced by quality scores in meta-analysis, and a hierarchical view of proposed solutions. Biostatistics. 2001; 2(4):463-471. [PubMed: 12933636]

4. Juni P, Witschi A, Block R, Egger M. The hazards of scoring the quality of clinical trials for metaanalysis. Journal of the American Medical Association. 1999; 282:1054-1060. [PubMed: 10493204]

5. Teixeira-Pinto A, Normand S-LT. Correlated bivariate continuous and binary outcomes: Issues and applications. Statistics in Medicine. 2009; 28:1753-1773. [PubMed: 19358234]

6. Neumann PJ, Kamae MS, Palmer JA. Medicare's national coverage decisions for technologies, 1997-2007. Health Affairs. 2008; 6:1620-1631. [PubMed: 18997221]

7. Lee PY, Alexander KP, Hammill BG, Pasquali SK, Peterson ED. Representation of elderly persons and women in published randomized trials of acute coronary syndromes. Journal of the American Medical Association. 2001; 286(6):708-713. [PubMed: 11495621]

8. Murthy VH, Krumholz HM, Gross C. Participation in clinical trials: Race-, sex- and age-based disparities. Journal of the American Medical Association. 2004; 291(22):2720-2726. [PubMed: 15187053]

9. Shea L, DuMouchel W, Bahamonde L. A meta-analysis of 16 randomised controlled trials to evaluate computer-based clinical reminder systems for preventive care in the ambulatory setting. Journal of the American Medical Informatics Association. 1996; 3(6):399-409. [PubMed: 8930856]

10. Lumley T. Network meta-analysis for indirect treatment comparisons. Statistics in Medicine. 2002; 21:2313-2324. [PubMed: 12210616]

11. Goodman SN. Towards evidence-based medical statistics 2: the bayes factor. Annals of Internal Medicine. 1999; 130(12):1005-1013. [PubMed: 10383350] 
Table 1

\section{MedCAC Process of Evaluation}

1 Overview: What evidence exists that a new medical item or service is effective and likely to improve health outcomes of Medicare beneficiaries? The quality of the evidence from different sources will vary, and the committee should weigh the evidence according to its quality.

2 Outcomes evaluated: How, compared to alternative or standard management approaches for the condition under review, does the intervention affect: quality of life; morbidity; mortality; diagnostic accuracy; and other health outcomes as appropriate, such as rehospitalizations.

3 Quality of evidence: Determine whether the scientific evidence is of adequate quality to draw conclusions about the effectiveness of the intervention in routine clinical use in the population of Medicare beneficiaries. This involves the following two questions:

a. How close are the effects measured in the study to their true value(s)? The degree to which the study result differs from the underlying truth is composed of two factors; chance and bias.

b. How applicable are the results to the Medicare population, in the settings in which they receive care? The studies are often conducted in settings that differ from those in which the typical Medicare beneficiary receives care.

4 Size of health effect and net health outcomes: Establish how the effectiveness of the new intervention compares to the effectiveness of established services and medical interventions. Is there is a net health benefit; does the magnitude of beneficial health effect outweigh the adverse health effects. 